MOTOR SYSTEMS

\section{Thumbs up}

Motor skills are easily acquired through practise, partially through the acquisition of a motor memory: a change in the cortical movement representation. The results of a new study, published in the Journal of Neuroscience, show that just observing an action can result in the formation of similar motor memories.

When applied over the primary motor cortex, transcranial magnetic stimulation (TMS) can evoke finger and thumb movements. The direction of such TMS-evoked thumb movements can be altered by the prior practise of unidirectional thumb movements: the direction of subsequent evoked thumb movements is changed to align with the direction of the practised movements. This change is thought to reflect a modification of the underlying movement representation in the primary motor cortex.
Katja Stefan and colleagues used the same paradigm to look at how simply observing an action might alter such movement representation. They first established a baseline measure for TMSevoked activity by applying TMS to the primary motor cortex, which resulted in involuntary thumb movements. Subjects then participated in either a physical training session, in which they practised voluntary thumb movements in a direction opposite to baseline TMSevoked thumb movements, or they simply observed an actor making the same movements on-screen, without any physical practise. One-third of subjects also

saw an actor making similar thumb movements on-screen, but this time in the same direction as the baseline TMS-evoked thumb movements. Finally, TMS-evoked thumb movements were measured again in all subjects and compared with the baseline movements in each individual.

Although the effect was smaller, observing a movement away from the baseline direction resulted in changes in evoked thumb movement similar to those caused by actual physical practise. In both cases, evoked thumb movements changed so that they were more similar to the observed or practised movement. These results suggest that observation alone might result in specific changes in motor representation. The formation of such motor memories may rely on the activity of mirror neurons, which respond both to action execution and observation.

Further studies are required to confirm the role of mirror neurons in the formation of such observation-based motor memories, and to identify the exact location at which these neurons are active. Such observational practise might also prove to be an effective rehabilitative treatment for patients with motor problems who are unable to carry out physical practise.

Charvy Narain, Associate Editor, Nature Neuroscience

\section{(4) References and links}

ORIGINAL RESEARCH PAPER Stefan, K. et al. Formation of motor memory by action observation. J. Neurosci. 25 , 9339-9346 (2005)

FURTHER READING Classen, J. et al. Rapid plasticity of human cortical movement representation induced by practice. J. Neurophysiol. 79,1117-1123 (1998)

\section{A new stop signal}

Failure of axonal regeneration in the adult mammalian CNS is partly due to the inhibitory effect of molecules associated with CNS myelin and the glial scar, but the underlying intracellular signalling mechanisms are not clear. Writing in Science, Koprivica and colleagues show that inhibition of regeneration is mediated by activation of the epidermal growth factor receptor (EGFR), and that EGFR inhibitors promote significant repair of injured optic nerve fibres.

The potent inhibitory effect of myelin and the glial scar on axonal growth can be easily studied in culture. When neurons are grown in the presence of either myelin or the active component of the glia scar, chondroitin sulphate proteoglycans (CSPGs), they will have little neurite arborization compared with their couterparts grown in control conditions. This easy assay has allowed Koprivica et al. to screen $\sim 400$ well characterized small molecules for their ability to reverse the inhibitory activity of myelin and CSPGs.
Surprisingly, several EGFR kinase inhibitors, including AG1478, PD168393 and Tarceva, enhanced neurite extension from dissociated rat cerebellar granule and dorsal root ganglion (DRG) neurons grown in the presence of either myelin or CSPGs. Moreoever, axonal growth of DRG neurons expressing a dominant-negative mutant form of EGFR was no longer blocked by myelin or CSPGs, indicating that EGFR kinase activity might be involved in the inhibition of regeneration.

This prompted the researchers to test the effect of myelin on EGFR phosphorylation in serum-starved cerebellar granule neurons. Both Nogo66 and oligodendrocyte myelin glycoprotein - myelin-associated inhibitory proteins - triggered rapid EGFR phosphorylation. This effect was not seen in neurons overexpressing a dominant-negative mutant form of $\mathrm{NgR}$, the common ligandbinding component of the receptor complexes, which indicates that $\mathrm{NgR}$ or its functional homologues might be necessary for EGFR activation.

Do EGFR inhibitors have an effect on nerve repair in vivo? To test this, Koprivica et al. crushed the optic nerve in adult mice and applied gelform containing PD168393 at the lesion site immediately after injury. Two weeks later, there was substantial axonal growth in animals treated with the EGFR inhibitor, resulting in a ninefold increase in the number of regenerating fibres $0.25 \mu \mathrm{m}$ beyond the injury site compared with the control.

This surprising finding has important therapeutic implications for patients with spinal or other CNS injuries. As one of the EGFR inhibitors, Tarceva, has been approved by the US Federal Drug Administration for the treatment of lung cancer, its efficacy in nerve repair may soon be tested in clinical trials.

Jane Qiu

(2) References and links

ORIGINAL RESEARCH PAPER Koprivica, V. et al. EGFR activation mediates inhibition of axon regeneration by myelin and chondroitin sulfate proteoglycans. Science 310, 106-110 (2005) FURTHER READING Filbin, M. T. Myelin-associated inhibitors of axonal regeneration in the adult mammalian CNS. Nature Rev. Neurosci. 4, 703-713 (2003)

WEB SITE

He's laboratory: http://www.hms.harvard.edu/dms/neuroscience/ fac_he.html 